\title{
1 Alpha-synuclein induces epigenomic dysregulation of glutamate signaling and
} 2 locomotor pathways

3 Samantha L. Schaffner BSc ${ }^{1}$, Zinah Wassouf $\mathrm{PhD}^{2,3 \pm}$, Diana F. Lazaro PhD², Mary Xylaki MSc ${ }^{2}$, Nicole

4 Gladish BSc${ }^{1}$, David T. S. Lin PhD ${ }^{1}$, Julia Maclsaac PhD', Katia Ramadori BSc ${ }^{1+}$, Julia M. Schulze-Hentrich

$5 \mathrm{PhD}^{4}$, Tiago F. Outeiro PhD ${ }^{2,3,5,6 *^{*}}$, and Michael S. Kobor PhD ${ }^{1 * *}$

$6 \quad{ }^{1}$ Department of Medical Genetics, Centre for Molecular Medicine and Therapeutics,

7 British Columbia Children's Hospital Research Institute, University of British Columbia,

8 Vancouver, BC, Canada

$9{ }^{2}$ Department of Experimental Neurodegeneration, Center for Nanoscale Microscopy and Molecular

10 Physiology of the Brain, Center for Biostructural Imaging of Neurodegeneration, University Medical

11 Center Goettingen, 37073, Göttingen, Germany

$12{ }^{3}$ German Centre for Neurodegenerative Diseases (DZNE), Göttingen, Germany

$13{ }^{4}$ Institute of Medical Genetics and Applied Genomics, University of Tübingen, Tübingen, Germany

$14{ }^{5}$ Max Planck Institute for Experimental Medicine, 37075 Göttingen, Germany

${ }^{6}$ Translational and Clinical Research Institute, Faculty of Medical Sciences, Newcastle University, Framlington Place, Newcastle Upon Tyne, NE2 4HH, UK

IPresent address: Wellcome Sanger Institute, Wellcome Genome Campus, Hinxton, Cambridgeshire, UK 'Present address: BC Cancer Genetics and Genomics Laboratory, 600 W 10th Ave \#3305, Vancouver, BC, Canada

${ }^{*}$ Corresponding Author: Michael S. Kobor

Email: $\underline{\text { msk@bcchr.ca }}$

Phone: +1 604-875-3803

Address: 950 W 28th Ave, Vancouver, BC, Canada, V5Z 4H4

${ }^{*}$ Corresponding Author: Tiago F. Outeiro

Email: touteir@gwdg.de

Phone: +49 (0)551-39-13544

Address: Waldweg 33, 37073 Göttingen, Germany

†These authors share equal senior authorship.

Running Title: Alpha-synuclein alters the DNA methylome

Key Words: Alpha-synuclein, epigenetics, DNA methylation, DNA hydroxymethylation, glutamate signaling

Financial Disclosure/Conflict of Interest: The authors declare that the research was conducted in the absence of any commercial or financial relationships that could be construed as a potential conflict of interest.

Funding: This research was conducted under a Transnational "Epigenomics of Complex Diseases" grant (CIHR EGM-141897, ANR-15-EPIG-0001, BMBF 01KU1503A). TFO is supported by the Deutsche Forschungsgemeinschaft (DFG, German Research Foundation) under Germany's Excellence Strategy EXC 2067/1- 390729940, and SFB1286 (Project B6). 


\section{Abstract}

47 Background: Mutations and multiplications in the gene encoding for alpha-synuclein are associated with Parkinson's disease (PD). However, not all individuals with alpha-synuclein variants develop PD, suggesting that additional factors are involved. We hypothesized that increased alpha-synuclein might alter epigenetic regulation of PD pathways.

Objectives: To identify genome-wide DNA methylation and hydroxymethylation changes induced by overexpression of two alpha-synuclein variants in human dopaminergic neurons, and to relate these to

53 the corresponding transcriptome.

54 Methods: We assessed DNA methylation and hydroxymethylation at $>850,000 \mathrm{CpGs}$ using the EPIC BeadChip in LUHMES cells differentiated to dopaminergic neurons. Control LUHMES neurons, LUHMES neurons overexpressing wild type alpha-synuclein, and LUHMES neurons overexpressing A30P alphasynuclein were compared. We used SMITE network analysis to identify functionally related genes with altered DNA methylation, DNA hydroxymethylation, and/or gene expression, incorporating LUHMES H3K4me1 ChIP-seq to delineate enhancers in addition to the default promoter and gene body regions. Results: Using stringent statistical thresholds, we found that increased expression of wild type or A30P mutant alpha-synuclein induced DNA methylation changes at thousands of CpGs and DNA hydroxymethylation changes at hundreds of CpGs. Differentially methylated sites in both genotypes were enriched for several processes including movement-associated pathways and glutamate signaling.

64 For glutamate and other signaling pathways (i.e. PDGF, insulin), this differential DNA methylation was also associated with transcriptional changes.

66 Conclusions: Our results indicated that alpha-synuclein altered the DNA methylome of dopaminergic 67 neurons, influencing regulation of pathways involved in development, signaling, and metabolism. This supports a role for alpha-synuclein in the epigenetic etiology of PD. 


\section{0 \\ Introduction}

71 Parkinson's disease (PD) is characterized pathologically by the presence of alpha-synuclein (aSyn)-rich

72 Lewy bodies in the brain. The function of aSyn is still unclear, as different cellular roles have been

73 reported, including SNARE complex assembly, regulation of neuronal differentiation, glucose levels, and

74 dopamine biosynthesis, as well as modulation of calmodulin activity/G-protein-coupled receptor kinase

75 activity $^{1}$. aSyn point mutations can impair one or several of these processes, disrupting neuronal health;

76 however, the mechanism leading to PD may differ according to the variant. For example, the A30P

77 mutation occurs in the membrane binding domain of aSyn, which may affect its ability to act as a

presynaptic chaperone and interact with membrane-bound receptors ${ }^{2}$. Although A30P aSyn is less likely

to aggregate than wild type (WT) aSyn, this mutation can still result in PD by loss of aSyn function².

81 aggregations and fibrils that impair synaptic function and can lead to neuronal death ${ }^{3}$. In familial PD

82 patients, duplications and triplications of SNCA have been reported ${ }^{4}$.

83 Although a clearer picture of the relationship between aSyn dysfunction and PD is forming, much

84 remains to be elucidated. For example, PD is variably penetrant even among individuals with pathogenic

85 SNCA mutations, suggesting that additional genes and the environment likely play a role in etiology ${ }^{4}$.

86 Since different aSyn mutations can lead to PD through different mechanisms, it is also important to

87 understand which aspects of these variants are unique and which are shared. Building on this, our group

88 and others have shown that expression of wild type or A30P mutant aSyn in human neurons can cause

89 transcriptional dysregulation of cell survival and DNA repair genes, which may be mediated by altered

90 histone $\mathrm{H} 3$ acetylation ${ }^{5-6}$. Additionally, altered DNA methylation (DNAm) patterns have been observed

91 both at the SNCA gene itself and genome-wide in blood, saliva, and brain of PD patients ${ }^{7-11}$.Taken 
92 together, this implies that genome-wide transcriptional and epigenetic dysregulation may be involved in

93 PD susceptibility and pathogenesis.

94 Epigenetic studies of PD provide the opportunity to assess genetic and environmental influences on

95 disease risk concurrently ${ }^{12-18}$. DNAm, which refers to the attachment of a methyl group to DNA (on the 5'

96 carbon of cytosine, frequently in the context of CpG dinucleotides), is a well-studied epigenetic mark

97 that changes during development and is influenced by genes and environment ${ }^{12-15,19}$. Although DNAm

98 patterns can be unrelated to mRNA expression patterns or laid down as a consequence of gene expression, DNAm can in some cases also impact transcription by altering the ability for transcription factors to bind to genes, changing chromosomal interactions, and influencing splicing ${ }^{12,20-23}$.This can alter regulation of pathways involved in disease susceptibility ${ }^{24-26}$. Additionally, DNAm patterns can also be useful as biomarkers to indicate aging, diseased states, or exposures regardless of association with transcription $27-33$.

Although DNAm is influenced by a myriad of factors that differ between individuals, the existing literature on DNAm in PD has primarily taken a case-control approach ${ }^{7-11}$. Additionally, the role of DNA hydroxymethylation (DNAhm), which accounts for up to $40 \%$ of all modified cytosines in brain tissue,

107 remains unclear ${ }^{34}$. DNAhm is an oxidized form of DNAm, and an intermediate of the DNA demethylation process; however, studies have suggested that DNAhm is also a stable, independent mark in brain ${ }^{35}$. The stability of DNAhm and its ability to influence transcription (by eliminating DNAm-protein 110 interactions, introducing DNAhm-protein interactions, altering chromatin state, and altering splicing)

111 suggest that it could impact neurological disease etiology ${ }^{36-37}$. It is important to distinguish DNAhm from 112 DNAm, since conventional bisulfite conversion techniques measure both DNAhm and DNAm in one 113 compound signal, which may bias interpretations ${ }^{38}$. DNAhm is stable in post-mortem formalin-fixed 114 tissue, and some initial studies have characterized DNAhm patterns in brains of deceased PD patients ${ }^{39}$. 115 Bulk DNAhm levels are unchanged in the cortex, substantia nigra, and brainstem of PD patients, while 
DNAhm levels and expression of TET2, the enzyme which forms this mark, are elevated in neurons from

117 prefrontal cortex of PD patients ${ }^{40-41}$. Importantly, DNAm and DNAhm patterns can be influenced by

118 genetics, and genetic alterations at loci such as SNCA influence PD susceptibility, which suggests that

119 incorporating a genetic lens into future epigenetic studies of PD will be important ${ }^{42-44}$. To date, only a

120 few studies have assessed the impact of genetic background on DNAm patterns in PD ${ }^{45-47}$.

121 One of the major challenges in human epigenetic studies of PD is accounting for the range of genetic,

122 environmental, and lifestyle factors that can influence DNAm and DNAhm ${ }^{42,48}$. Furthermore, using

123 postmortem tissue to study PD also typically presents the limitation of advanced disease. Model systems

124 such as rodents or cell culture help to address these issues, providing a context where both genetics and

125 environment can be tightly controlled, and the opportunity to examine early impacts of neurological

126 disease in brain.

127 In this study, we characterized the influence of two molecularly distinct aSyn variants on the DNA

128 methylome and hydroxymethylome of human dopaminergic neurons. We profiled genome-wide DNAm

129 and DNAhm patterns in three groups of Lund human mesencephalic (LUHMES) cells differentiated into

130 dopaminergic neurons: control LUHMES, LUHMES overexpressing WT aSyn at levels similar to those

131 seen in SNCA multiplication carriers, and LUHMES overexpressing A3OP aSyn. We additionally integrated

132 our DNAm and DNAhm data with transcriptomic data from the same cells, incorporating H3K4me1 ChIP-

133 seq to score DNA(h)m changes across gene regulatory features ${ }^{6}$. WT and A30P aSyn expression were

134 associated with thousands of DNA(h)m changes, particularly affecting genes that regulate locomotory

135 and glutamate signaling pathways. This suggested that familial PD-associated aSyn mutations have

136 widespread epigenomic effects, and may contribute to molecular and transcriptional dysregulation

137 associated with PD etiology and heterogeneity. 


\section{Materials and Methods}

\section{Generation of aSyn expressing LUHMES cells}

141 LUHMES cells were a gift from Prof. Marcel Leist and were cultured and differentiated as previously

142 described ${ }^{6}$ (Supplementary Methods). In brief, full-length human WT or A30P aSyn cDNA was cloned

143 into a biscistronic lentiviral vector under a chicken beta-actin promoter, and transiently transfected into

$144293 T$ cells. Proliferating LUHMES were infected with equimolar amounts of IRES-GFP, WT aSyn-IRES-GFP

145 or A30P aSyn-IRES-GFP lentivirus. Infected cells were selected by FACS, and differentiated for eight days ${ }^{6}$

146 (Supplementary Methods).

EPIC BeadChip data generation and bioinformatic analysis

DNA was extracted from cell pellets (see Supplementary Methods), and $750 \mathrm{ng}$ per sample of bisulfite

149 (BS)- or oxidative bisulfite (oxBS)-converted DNA was run on Infinium HumanMethylationEPIC (EPIC)

BeadChips (Illumina Inc.) according to the manufacturer's instructions, producing data for 853,307 CpG

and 2,880 CNG sites.

Beta values were generated from raw intensity signals using GenomeStudio software (Illumina) and

exported into R 3.6 for data analysis. SNP control probes, cross-hybridizing probes, and low-quality

154 probes were removed, leaving 813,589 EPIC probes in the final dataset ${ }^{49-50}$. oxBS and BS data were

155 normalized separately with dasen, and batch effects were removed with ComBat ${ }^{51}$. Hydroxymethylation

$156(\mathrm{hmC})$ values were calculated by subtraction, and a detectability threshold of $3.6 \%$ was set using the

$15795 \%$ quantile of resulting negative values ${ }^{52}$. See Supplementary Methods for more detailed data

158 processing information.

Differential methylation between control $(n=7)$, WT aSyn $(n=8)$, and A30P aSyn $(n=8)$ cells was calculated 
161 considered significant at FDR $<=0.05$ and $\mid$ delta beta $\mid>=0.05$ (calculated by subtracting the mean beta

162 value across all replicates of the group in question from the reference group, e.g. WT aSyn beta - Control

163 beta). The skewness.norm.test function from the normtest $\mathrm{R}$ package was used to assess delta beta

164 skewness, with 1000 Monte Carlo simulations ${ }^{54}$.

\section{Pyrosequencing}

See Supplementary Methods.

\section{Gene ontology enrichment}

168 Genes were assigned to CpGs if they belonged to the longest UCSC RefGene transcript annotated to 169 each site, resulting in 30,325 unique coding- and non-coding gene annotations for the DNAm dataset 170 and 23,190 unique gene annotations for the DNAhm dataset. Gene ontology enrichment was performed

171 with the over-representation (ORA) method in ermineR 1.0.1.9, using differentially methylated genes 172 identified with the effect size and significance thresholds previously mentioned as input ${ }^{55}$. Genes that 173 were differentially methylated or hydroxymethylated in both WT aSyn and A30P aSyn cells in either

174 direction were removed from the input list and analyzed separately.

\section{Chromatin immunoprecipitation sequencing (ChIP-seq) and peak calling}

176 See Supplementary Methods.

\section{Multi-omic integration}

178 DNAm, DNAhm, and RNA-seq data were integrated using SMITE in R 3.6, with adjusted p-values as

179 significance input, delta betas as effect size input for DNAm and DNAhm, and log2FC values for RNA-

$180 \operatorname{seq}^{56}$ (Supplementary Methods). H3K4me1 ChIP-seq peaks were used to define enhancers. The following 181 weights were applied during scoring: expression, 0.4; enhancer DNAm, 0.125; promoter DNAm, 0.125; 
182

183

184

185

body DNAm, 0.1; enhancer DNAhm, 0.125; promoter DNAhm, 0.125; body DNAhm, 0.1. Gene scores were annotated to a REACTOME protein-protein interaction network.

\section{Results}

\section{aSyn overexpression altered genome-wide DNAm patterns in dopaminergic neurons}

In order to evaluate the epigenome-wide impacts of expressing aSyn in dopaminergic neurons, we assessed DNAm differences between control, WT aSyn, and A30P aSyn cells at 813,589 EPIC probes. We selected an effect size threshold of $\mid$ delta beta $\mid>=0.05$ to be well above technical noise (maximum 2.2\% RMSE between technical replicates). Firstly, we sought to confirm whether overexpression of aSyn was associated with reduced DNAm at the first intron of SNCA, as previously reported in PD patients ${ }^{10-11}$; this was true for both aSyn genotypes (Fig. 1). We next assessed genome-wide DNAm changes induced by overexpression of each aSyn variant (Fig. 2). When WT aSyn cells were compared to control, 18,521 sites had decreased DNAm and 10,812 sites had increased DNAm (padj <=0.05, Fig. 2A, Table S2). In A30P aSyn cells, 3,091 probes had decreased DNAm compared to control while 3,438 probes had increased DNAm (Fig. 2A, Table S3). When compared to WT aSyn cells, A30P aSyn cells had 3,014 sites with decreased DNAm and 6,666 sites with increased DNAm (Fig. 2A, Table S4). DNAm changes in all comparisons were significantly skewed to one direction, and the bias in effect direction was seen across all genomic features $(p<2.2 \mathrm{e}-16$, Table S5). To confirm an example from our findings by pyrosequencing, we selected a region of the TUBA8 gene that included the top two probes with the largest change in DNAm across both genotypes; differential DNAm at these two positions, one additional position on the array, and four positions not on the array were all confirmed (Fig. S1, Table S8). 

GO biological processes were enriched in differentially methylated sites shared between the control vs.

The proportion of differentially methylated probes shared across all comparisons and any two of three comparisons was larger than expected by chance, and in most cases, WT aSyn cells had more pronounced differences from controls than A30P aSyn cells (Fig. 2B, padj $<0.005,10,000$ permutations; Fig. S2). Interestingly, WT and A30P aSyn overexpression primarily affected CpGs outside of genes; however, trends for differentially methylated probe feature enrichment differed between genotypes for TSS1500 regions and CpG North Shores (Fig 2C-D, both enriched in WT cells and depleted in A30P cells).

\section{aSyn overexpression altered genome-wide DNAhm patterns to a lesser degree than DNAm patterns}

After assessing genome-wide DNAm alterations, we examined differential DNAhm at 233,440 sites that passed our detection threshold of $3.6 \%$ (calculated according to the $95 \%$ quantile of negative $\mathrm{hmC}$ values after subtraction; see Methods). Fewer DNAhm changes for each comparison were observed as compared to DNAm, most of which were increases; these increases in DNAhm were significant when tested for skewness ( $p<2.2 \mathrm{e}-16$, Fig. 3, Fig. S3, Tables S9-S11). The overlap of 16 CpG sites between control vs. WT aSyn and control vs. A30P aSyn was not higher than expected by chance (Fig. 3B, padj > 0.05, 10,000 permutations).

\section{WT and A30P aSyn altered DNAm at movement-associated and glutamate signaling pathway genes}

To identify possible functional consequences associated with the DNAm and DNAhm changes in each genotype, we performed gene ontology enrichment analysis on all differentially methylated or hydroxymethylated genes within each comparison using over-representation analysis in ermine $\mathrm{R}^{57} .34$ WT aSyn and control vs. A30P aSyn comparisons (Fig. 4A, padj < 0.05). $442 \mathrm{CpG}$ sites were annotated to 
the top GO term, "locomotory behavior," and differentially methylated in at least one genotype (420 sites in WT aSyn cells and 89 sites in A30P aSyn cells, Fig. 4B-E). 129 differentially methylated sites were annotated to the second-highest ranked GO term, "glutamate receptor signaling pathway" (127 sites in WT aSyn cells and 18 sites in A30P aSyn cells, Fig. S5). No significant multifunctionality-corrected enrichments were observed for differentially methylated or hydroxymethylated sites in any of the other groups.

\section{aSyn impacted epigenetic and transcriptional regulation of glutamate, NOTCH, insulin, PDGF, and SHH}

\section{signaling network genes}

We next queried genes and pathways that associated with changes in both the epigenome and the transcriptome in WT and A30P aSyn cells. These loci represent candidates where DNAm and/or DNAhm may be regulating gene expression, and it is important to identify them in order to understand the molecular etiology of PD. Firstly, we compared the DNAm and DNAhm hits found in this study against previously identified differentially expressed genes ${ }^{6}$ (863 genes in WT aSyn cells and 1,315 genes in A30P aSyn cells, $|\log 2 \mathrm{FC}|>0.5$ and $p a d j<0.01)$. Out of 10,798 genes with data for all three modifications in WT aSyn cells, seven displayed differential DNAm, DNAhm, and expression, including an ionotropic glutamate receptor (GRIK2; Table S15). In A30P cells, two out of 10,831 genes had concurrent changes to these modifications (TSPEAR and SGPP2, Table S16). The relationship between DNAm, DNAhm, and expression varied depending on the gene and the CpG site in question (Tables S15-S16).

We additionally employed SMITE, which captures modules of functionally related genes with changes to at least one of either DNAm, DNAhm, or gene expression in each comparison ${ }^{56}$. We included H3K4me1 ChIP-seq data from the same LUHMES in our SMITE workflow, allowing us to consider DNAm and DNAhm changes at enhancers (in addition to default promoter and gene body regions), and weighted 
248 the importance of each modification (expression: 0.4, DNAm: 0.35, DNAhm: 0.25). In WT aSyn cells, 18

249 modules were identified (Table S17, Fig. S7). In A30P aSyn cells, 24 modules were identified (Table S18,

250 Fig. S8).

251 Glutamate receptor signaling modules discovered using SMITE stood out with more concurrent changes

252 to DNAm, DNAhm, and transcription in both genotypes. Two modules centered around GRIK2 were each

253 significantly altered in WT and A30P cells, matching with the direct comparison of differentially

254 (hydroxy)methylated versus differentially expressed genes. There was a negative correlation between

255 GRIK2 promoter DNAm and mRNA levels in both genotypes (Fig. 5).

\section{Discussion}

258 Elucidating the mechanisms by which PD develops and progresses in different individuals is paramount

259 to developing preventative strategies and treatments. Here, we explored whether DNAm and DNAhm

260 contributed to interindividual differences in PD etiology among carriers of aSyn variants. We assessed

261 the impact of overexpressing WT or A30P aSyn on genome-wide DNAm and DNAhm patterns in

262 dopaminergic neurons, the primary cell type affected in PD. We found that overexpressing either aSyn

263 variant induced thousands of DNAm changes and hundreds of DNAhm changes in pathways related to

264 PD and neurodegeneration, and that WT aSyn particularly impacted glutamate receptor signaling genes

265 on the epigenetic and transcriptional level. Distinct characteristics of each aSyn protein may explain why

266 both similar and unique effects on DNA(h)m were observed. This study enhances our understanding of

267 the wide-ranging genomic impacts of different aSyn forms and illuminates further possible molecular

268 mechanisms for PD. 
The dysregulation of locomotor behaviour pathway genes was reassuring and indicative of the relevance and validity of our approach since this is one of the major systems affected in PD. Excessive

271 glutamatergic transmission can also play a role in PD, causing excitotoxicity in dopaminergic neurons ${ }^{58}$.

272 Preclinical studies have implicated glutamate receptors as therapeutic targets for PD, and regulation of

273 glutamate signaling genes may be influenced by lifestyle factors such as dietary exposure to neurotoxins

274 or tea polyphenols ${ }^{16,59-61}$. Our results thus provide evidence from an epigenetic perspective to expand

275 the existing research on the role of glutamate signaling in PD etiology and prevention, and add a role for

276 aSyn in this pathway.

277 Aside from these core similarities in the pathways affected by each aSyn variant, we also saw unique

278 effects of WT and A30P aSyn on the DNA methylome and hydroxymethylome. Broadly, many more

279 DNAm and DNAhm changes were seen in WT aSyn cells as compared with A30P cells. This was surprising

280 in light of our previous work, which identified that A30P cells are affected to a greater extent on the

281 transcriptional level ${ }^{6}$. It was also unexpected that almost no changes to non-CpG methylation were observed with aSyn overexpression (one differentially methylated CH probe in WT vs A3OP aSyn cells, data not shown) despite known enrichment of non-CpG methylation in neurons ${ }^{62-63}$. Several mechanisms might explain this. Firstly, DNA damage occurs to a greater extent in WT aSyn LUHMES neurons than in A30P aSyn LUHMES neurons, and it is possible that some DNAm changes in WT cells are a reflection of this damage and/or subsequent repair attempts ${ }^{6,64}$. Following DNA damage, protective mechanisms in areas of active transcription maintain DNA repair and restore epigenetic modifications; this may also explain why we observed more DNAm changes in intergenic regions and less in intragenic regions than expected by chance in both genotypes ${ }^{64-65}$.

291 in WT cells. WT aSyn could influence DNAm through various means, such as binding to membrane- 
assembly at presynaptic membranes, which could allow neurotransmitters to activate signaling cascades

294 at the postsynaptic neuron; and binding to DNA ${ }^{3,66}$. The A30P mutation prevents aSyn from binding to membranes, removing some of these avenues ${ }^{4}$.

WT aSyn can sequester Dnmt1 from the nucleus in mice, resulting in global loss of DNA methylation in the brain ${ }^{67}$. If there is a difference in DNMT1 sequestration between aSyn variants, this could reduce the capacity for maintenance of DNAm in a non-specific manner. aSyn aggregation - which is more likely with the WT protein - has also been demonstrated to increase this sequestration ${ }^{68}$. Although A3OP aSyn is more likely to localize to the nucleus than WT aSyn, the ability of A30P protein to sequester Dnmt1 has not been assessed ${ }^{66,68-69}$. In addition to influencing the DNAm loss in WT cells and gain in A30P cells, this potential difference in nuclear aSyn could also affect the amount of DNAm at transcription-

303 associated regions, due to its ability to bind DNA ${ }^{66,68-69}$.

In contrast to DNAm, patterns of differential hydroxymethylation were similar between aSyn genotypes, and fewer DNAhm changes were seen at our statistical thresholds. This was expected due to the fetal origin of the LUHMES cell line ${ }^{36,70}$. The opposite direction of change seen for DNAm and DNAhm at the same loci was also expected, since DNAm must be oxidized in order for DNAhm to form ${ }^{71}$. We are unable to determine from our study whether this DNAhm was a transient intermediate or represents a

309 stable epigenetic mark; however, correlating DNAhm patterns with gene expression levels provided some insight on loci where DNAhm was associated with transcription ${ }^{35}$.

311 It is important to understand the interplay between DNAm, DNAhm, and gene expression to gain insight

312 into the molecular etiology of PD. Changes to DNAm may be unrelated to transcription, or precede or

313 follow changes to transcription ${ }^{12,20-21}$. In the former case, DNAm may represent a target for modulation

314 of gene expression in order to prevent, for example, upregulation of glutamate signaling genes in

315 individuals with aSyn multiplications. In the latter case, DNAm is more likely to represent a biomarker of 
expression. The relatively low correlation between DNA(h)m changes and expression changes in this

317 study ( $<0.1 \%$ of genes in either genotype) agrees with previous literature, which has reported

correlations with expression at only $0.6-8 \%$ of $\mathrm{CpG}$ sites in blood and up to $0.3 \%$ of $\mathrm{CpG}$ sites in brain ${ }^{72-76}$.

However, the few loci that did have transcriptional changes correlated with DNA(h)m changes may still

represent either functionally relevant targets for PD or potential biomarkers for neurodegeneration-

relevant pathways.

Although this study provided insights into the epigenomic impact of aSyn overexpression, several

limitations should be considered. The SNCA transgene integrated at random positions in the genome,

327 influence DNAm ${ }^{77-79}$. Our LUHMES replicates were obtained from a range of passages and culture dishes, 
bioRxiv preprint doi: https://doi.org/10.1101/2021.06.12.448150; this version posted June 12, 2021. The copyright holder for this preprint

(which was not certified by peer review) is the author/funder, who has granted bioRxiv a license to display the preprint in perpetuity. It is made available under aCC-BY-NC-ND 4.0 International license.

340 with increased expression or mutations in aSyn may be reversible with environment and/or lifestyle

341 factors.

342

343 Author Roles

344 1. Research project: A. Conception, B. Organization, C. Execution; 2. Statistical Analysis: A. Design, B.

345 Execution, C. Review and Critique; 3. Manuscript: A. Writing of the first draft, B. Review and Critique.

346 S.L.S: 1A, 1B, 1C, 2A, 2B, 3A.

347 Z.W.: 1B, 1C, 2A, 2B, 3B.

348 D.F.L.: 1B, 1C, 3B.

349 M.X.: 1B, 1C, 3B.

$350 \quad$ N.G.: 2C, 3B.

351 D.T.S.L: 1C, 3B.

352 J.M.: 1C, 3B.

353 K.R.: 1C, 3B.

354 J.M.S.H.: 1A, 1B, 3B.

355 T.F.O: 1A, 1B, 3B.

356 M.S.K.: 1A, 1B, 2C, 3B.

357 


\section{$359 \quad$ Financial Disclosure}

SLS was supported by a Fredrick Banting and Charles Best Canada Graduate Scholarships (CGS-D) award from the Canadian Institutes of Health Research. ZW was supported by the German Center for Neurodegenerative Diseases (DZNE) in Goettingen, Germany. DFL received funding from ParkinsonFonds. MX received support from SFB1286 (Project B6). NG was funded by the Sunny Hill Health Centre BC Leadership Chair in Early Childhood Development Endowment Trust Fund. DTSL received funding from the National Institutes of Health / Research Project Grant (R01; Sponsor Identifier: 19-A0-00-1003237, 114579), and the Allergy, Genes and Environment Network (AllerGen) Networks of Centres of Excellence (NCE) (Sponsor Identifier: 12GxE2). JM was supported by Canadian Institutes of Health Research (CIHR) / Project Scheme: 2016 1st Live Pilot (Sponsor Identifier: PJT148925) and Canadian Institutes of Health Research (CIHR) / Team Grant: ERA-HDHL Call for Transnational Research Proposals: "Nutrition and the Epigenome" (Sponsor Identifier: NTE-160943). KR was funded by the BC Cancer Genetics and Genomics Laboratory. JMSH received a fellowship from the Brigitte-Schlieben-Lange program that is supported by the Ministry of Science, Research and the Arts Baden-Württemberg, Germany. TFO was supported by the Deutsche Forschungsgemeinschaft (DFG, German Research Foundation) under Germany's Excellence Strategy (EXC 2067/1- 390729940) and by SFB1286 (Project B6). MSK was supported by a Canada Research Chair Tier 1 in Social Epigenetics, the Sunny Hill BC Leadership Chair in Child Development, and the Canadian Institute for Advanced Research. He received grant funding from Canadian Institutes of Health Research, Natural Sciences and Engineering Research Council of Canada, National Institutes of Health, National Science Foundation, Genome Canada, Networks of Centres of Excellence, R. Howard Webster Foundation, and UBC VP Research \& Innovation. 


\section{2 \\ References}

383

1. Emamzadeh FN. Alpha-synuclein structure, functions, and interactions. J Res Med Sci 2016; 9: 21-29.

2. Jo E, Fuller N, Rand RP, St George-Hyslop P, Fraser PE. Defective membrane interactions of familial Parkinson's disease mutant A30P alpha-synuclein. J Mol Biol 2002; 315: 799-807.

3. Volpicelli-Daley LA, Luk KC, Patel TP, Tanik SA, Riddle DM, Stieber A, Meaney DF. Exogenous $\alpha$ Synuclein Fibrils Induce Lewy Body Pathology Leading to Synaptic Dysfunction and Neuron Death. Neuron 2011; 72(1): 57-71.

4. Lesage S, Brice A. Parkinson's disease: from monogenic forms to genetic susceptibility factors. Hum Mol Genet 2009; 18(1): R48-R59.

5. Baptista MJ, O'Farrell C, Daya S, et al. Co-ordinate transcriptional regulation of dopamine synthesis genes by alpha-synuclein in human neuroblastoma cell lines. J Neurochem 2003; 85(4): 957-968.

6. Paiva I, Pinho R, Pavlou MA, et al. Sodium butyrate rescues dopaminergic cells from alpha-synucleininduced transcriptional deregulation and DNA damage. Hum Mol Genet 2017; 26(12): 2231-2246.

7. Masliah E, Dumaop W, Galasko D, Desplats P. Distinctive patterns of DNA methylation associated with Parkinson disease: identification of concordant epigenetic changes in brain and peripheral blood leukocytes. Epigenetics 2013; 8(10): 771-781.

8. Chuang Y, Paul KC, Bronstein J, Bordelon Y, Horvath S, Ritz B. Parkinson's disease is associated with DNA methylation levels in human blood and saliva. Genome Med 2017; 9(1): 76.

9. Rawlik K, Rowlatt A, Tenesa A. Imputation of DNA Methylation Levels in the Brain Implicates a Risk Factor for Parkinson's Disease. Genetics 2016; 204(2): 771-781. 

disease patients in vivo and in vitro. Mov Disord 2015; 30(13): 1794-1801. levels of alpha-synuclein intron 1 in the aging brain. Neurobiol Aging 2015; 36(12): 3334.e7-3334.e11. Methylation in Human Brain. Am J Hum Genet 2010; 86(3): 411-419. variation in neonate DNA methylomes. Genome Res 2014; 24(7): 1064-1074.

411 across the human life course. Genome Biol 2016; 17(61).

412 15. Wang H, Lou D, Wang Z. Crosstalk of Genetic Variants, Allele-Specific DNA Methylation, and

413 Environmental Factors for Complex Disease Risk. Front Genet 2019; 9(695).

414 16. Vallegra CL, Zhang F, Fowdar J, et al. Analysis of DNA methylation associates the cystine-glutamate 415 antiporter SLC7A11 with risk of Parkinson's disease. Nat Comm 2020; 11(1238): 1-10.

416 17. Go RCP, Corley MJ, Ross GW, et al. Genome-wide epigenetic analyses in Japanese immgigrant 417 plantation workers with Parkinson's disease and exposure to organchlorines reveal possible involvement 418 of glial genes and pathways involved in neurotoxicity. BMC Neuroscience 2020; 21(31): 1-18.

419 18. Paul KC, Horvath S, Del Rosario I, Bronstein JM, Ritz B. DNA methylation biomarker for cumulative 420 lead exposure is associated wtih Parkinson's disease. Clin Epigenet 2021; 13(59): 13-16.

421 19. Lim IY, Lin X, Karnani N. Implications of Genotype and Environment on Variation in DNA Methylation. 422 2017, In: Patel V, Preedy V. Handbook of Nutrition, Diet, and Epigenetics. 2017; 1-20. 
423 20. Lee KWK, Pausova Z. Cigarette smoking and DNA methylation. Front Genet 2013; 4: 132.

424 21. Lim U, Song MA. Dietary and lifestyle factors of DNA methylation. Methods Mol Biol 2012; 863: 359-

425376.

426 22. Jones PA. Functions of DNA methylation: Islands, start sites, gene bodies and beyond. Nat Rev Genet

$427 \quad 2012 ; 13(7): 484-492$

428 23. Zhang L, Xie WJ, Liu S, Meng L, Gu C, Gao YQ. DNA Methylation Landscape Reflects the Spatial

429 Organization of Chromatin in Different Cells. Biophysical Journal 2017; 113: 1395-1404.

430

24. Jin Z, Liu Y. DNA methylation in human diseases. Genes \& Diseases 2018; 5(1): 1-8.

431 25. Lappalainen T, Greally J. Associating cellular epigenetic models with human phenotypes. Nat Rev

432 Genet 2017; 18(7): 441-451.

433 26. Landgrave-Gómez J, Mercado-Gómex R, Guevera-Guzmán R. Epigenetic mechanisms in neurological

434 and neurodegenerative diseases. Front Cell Neurosci 2015; 9(58): 1-11.

435 27. Fransquet PD, Lacaze P, Saffery R, McNeil J, Woods R, Ryan J. Blood DNA methylation as a potential

436 biomarker of dementia: A systematic review. Alzheimer's and Dementia 2018; 14(1): 81-103.

437 28. Garagnani P, Bacalini MG, Pirazzini C, et al. Methylation of ELOV2 gene as a new epigenetic marker

438 of age. Aging Cell 2012; 11(6): 1132-1134.

439 29. Shenker NS, Ueland PM, Polidoro S, et al. DNA Methylation as a Long-term Biomarker of Exposure to

440 Tobacco Smoke. Epidemiology 2013; 25(5): 712-716.

441 30. Levenson VV. DNA methylation as a universal biomarker. Expert Review of Molecular Diagnostics $442 \quad 2010 ; 10(4): 481-488$. 
443 31. Salameh Y, Bejaoui Y, El Hajj N. DNA Methylation Biomarkers in Aging and Age-Related Diseases.

$444 \quad$ Front Genet 2020; 11(171): 1-11.

32. Horvath S. DNA methylation age of human tissues and cell types. Genome Biol 2013; 14(10): R115.

33. Levine ME, Lu AT, Quach A, et al. An epigenetic biomarker of aging for lifespan and healthspan. Aging

$447 \quad 2018 ; 10(4): 276162$.

448 34. Kinde B, Gabel HW, Gilbert CS, Griffith EC, Greenberg ME. Reading the unique DNA methylation

449 landscape of the brain: Non-CpG methylation, hydroxymethylation, and MeCP2. PNAS 2015; 112(22):

$450 \quad 6800-6806$.

451 35. Bachman M, Uribe-Lewis S, Yang X, Williams M, Murrell A, Balasubramanian S. 5-

452 Hydroxymethylcytosine is a predominantly stable DNA modification. Nat Chem 2014; 6: 1049-1055.

453 36. Szulwach KE, Li X, Li Y, et al. 5-hmC-mediated epigenetic dynamics during postnatal

454 neurodevelopment and aging. Nat Neurosci 2012; 14(12): 1607-1616.

455 37. Kumar S, Chinnusamy V, Mohapatra T. Epigenetics of Modified DNA Bases: 5-Methylcytosine and

456 Beyond. Frontiers in Genetics 2018; 9(640): 1-14.

457 38. Nestor C, Ruzov A, Meehan RR, Dunican DS. Enzymatic approaches and bisulfite sequencing cannot 458 distinguish between 5-methylcytosine and 5-hydroxymethylcytosine in DNA. BioTechniques 2010; 48(4):

$459 \quad 317-319$. 
463 40. Kaut O, Kuchelmeister K, Moehl C, Wüllner U. 5-methylcytosine and 5-hydroxymethylcytosine in

464 brains of patients with multiple system atrophy and patients with Parkinson's disease. J Chem

465 Neuroanat 2019; 96: 41-48.

466 41. Marshall LL, Killinger BA, Ensink E, et al. Epigenomic analysis of Parkinson's disease neurons

467 identifies Tet2 loss as neuroprotective. Nat Neurosci 2020; 23: 1203-1214.

42. Spiers H, Hannon E, Schalkwyk LC, Bray NJ, Mill J. 5-Hydroxymethylcytosine Is Highly Dynamic Across

469 Human Fetal Brain Development. BMC Genomics 2017; 18(1): 1-14.

470 43. Cheung WA, Shao X, Morin A, et al. Functional variation in allelic methylomes underscores a strong

471 genetic contribution and reveals novel epigenetic alterations in the human epigenome. Genome Biol

$472 \quad 2017 ; 18(1): 1-21$.

473 44. Klein C, Westenberger A. Genetics of Parkinson's Disease. CSH Persp Med 2012; 2(1): a008888.

474 45. International Parkinson's Disease Genomics Consortium, Wellcome Trust Case Control Consortium. A

475 Two-Stage Meta-Analysis Identifies Several New Loci for Parkinson’s Disease. PLoS Genet 2011; 7(6):

$476 \mathrm{e} 1002142$.

477 46. Nalls M, Pankratz C, Lill C, et al. Large-scale meta-analysis of genome-wide association data identifies

478 six new risk loci for Parkinson's disease. Nat Genet 2014; 46(9): 989-993.

479 47. Kia DA, Zhang D, Guelfi S, et al. Identification of Candidate Parkinson Disease Genes by Integrating

480 Genome-Wide Association Study, Expression, and Epigenetic Data Sets. JAMA Neurol 2021; 78(4): 464-

481472.

482 48. Kochmanski J, Bernstein Al. The Impact of Environmental Factors on 5-Hydroxymethylcytosine in the 483

Brain. Curr Envtl Health Rep 2020; 7(2): 109-120. 

microarray for whole-genome DNA methylation profiling. Genome Biol 2016; 17(208). preprocessing Illumina 450K methylation array data. BMC Genomics 2013; 14(293). Other Unwanted Variation in High-Throughput Experiments. Bioinformatics 2012; 28(6): 882-883. cerebellum. Genome Biol 2016; 17(27). analyses for RNA-sequencing and microarray studies. Nucleic Acids Res 2015; 43(7): e47. project.org/web/packages/normtest/normtest.pdf. 
59. Johnson KA, Conn PJ, Niswender CM. Glutamate receptors as therapeutic targets for Parkinson's

506

507

508

509

510

511

512

513

514

515

516

517

518

519

520

521

522

523

524 disease. CNS Neurol Disord Drug Targets 2009; 8(6): 475-491.

60. Cox PA, Davis DA, Mash DC, Metcalf JS, Banack SA. Dietary exposure to an environmental toxin triggers neurofibrillary tangles and amyloid deposits in the brain. Proc Biol Sci 2016; 283(20152397).

61. Chen S, Wang Z, Ma Y, Zhang W, Lu J, Liang Y, Zheng X. Neuroprotective Effects and Mechanisms of Tea Bioactive Components in Neurodegenerative Diseases. Molecules 2018; 23(3): 512.

62. Lister E, Mukamel E, Nery J, et al. Global Epigenomic Reconfiguration During Mammalian Brain Development. Science 2013; 341(1237905): 692-643.

63. Farré P, Jones MJ, Meaney MJ, Emberly E, Turecki G, Kobor MS. Concordant and discordant DNA methylation signatures of aging in human blood and brain. Epigenetics and Chromatin 2015; 8(1): 1-17.

64. Bernstein C, Bernstein H. The Role of DNA Repair and the Epigenetic Markers Left after Repair in Neurologic Functions, Including Memory and Learning. In: Mognato M. DNA Repair - An Update. 2019.

65. Huang Y, Gu L, Li G. H3K36me3-mediated mismatch repair preferentially protects actively transcribed genes from mutation. JBC 2018; 293(20): 7811-7823.

66. Kontoploulos E, Parvin JD, Feaney MB. Alpha-synuclein Acts in the Nucleus to Inhibit Histone Acetylation and Promote Neurotoxicity. Hum Mol Genet 2006; 15(20): 3012-3023.

67. Desplats P, Spencer B, Coffee E, et al. Alpha-Synuclein Sequesters Dnmt1 from the Nucleus. JBC 2011; 286(11): 9031-9037.

68. Irizarry RA, Ladd-Acosta C, Wen B, et al. The human colon cancer methylome shows similar hypoand hypermethylation at conserved tissue-specific CpG island shores. Nat Genet 2009; 41(2): 178-186. 

large-scale generation of post-mitotic neurons from the human LUHMES cell line. J Neurochem 2011; in functional demethylation of expressed genes. PNAS 2017; 114(37): E7812-E7821. variable DNA methylation in a human community cohort. PNAS 2012; 109(Suppl 2): 17253-17260. quantification of the effects of DNA methylation on human gene regulation. eLife 2018; 7: e37513.

537 expression in human monocytes and T cells. Nat Comm 2015; 5: 5366.

538 75. Chen C, Zhang C, Cheng L, et al. Correlation between DNA methylation and gene expression in the 539 brains of patients with bipolar disorder and schizophrenia. Bipolar Disord 2014; 16(8): 790-799.

540 76. Price AJ, Collado-Torres L, Ivanov NA, et al. Divergent neuronal DNA methylation patterns across

541 human cortical development reveal cirtical periods and a unique role of $\mathrm{CpH}$ methylation. Genome Biol $542 \quad 2019 ; 20(196)$.

543 77. Weber M, Davies JJ, Wittig D, Oakeley EJ, Haase M, Lam WL, Schübeler D. Chromosome-wide and 544 promoter-specific analyses identify sites of differential DNA methylation in normal and transformed 545 human cells. Nat Genet 2005; 37(8): 853-862. 
bioRxiv preprint doi: https://doi.org/10.1101/2021.06.12.448150; this version posted June 12, 2021. The copyright holder for this preprint (which was not certified by peer review) is the author/funder, who has granted bioRxiv a license to display the preprint in perpetuity. It is made available under aCC-BY-NC-ND 4.0 International license.

546 78. de Boni L, Gasparoni G, Haubenreich C, et al. DNA methylation alterations in iPSC- and hESC-derived

547 neurons: potential implications for neurological disease modeling. Clin Epigenet 2018; 10(13).

548 79. de Boni L, Wüllner U. Epigenetic Analysis in Human Neurons: Considerations for Disease Modeling in

549 PD. Front Neurosci 2019; 13(276).

550

551 

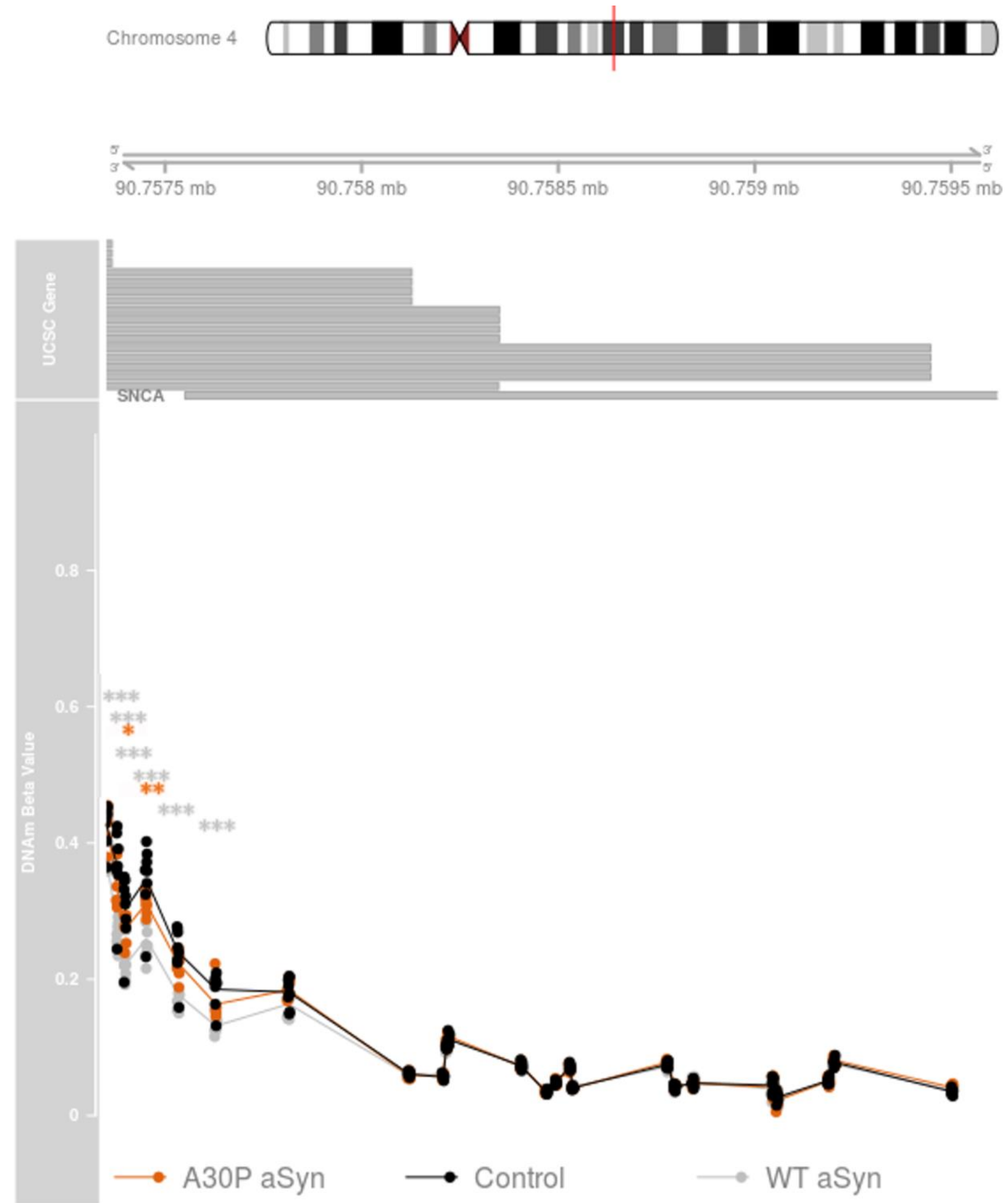

553 Figure 1. aSyn overexpression was associated with decreased DNAm at the SNCA 5' UTR. Top: UCSC 554 hg19 coordinates are shown; grey boxes represent SNCA transcripts. Bottom: DNAm beta values are 555 shown for each sample, coloured by genotype. Black: control cells; pale grey: WT aSyn cells; orange: 556 A30P aSyn cells. * padj $<0.05, * *$ padj $<0.005, * * *$ padj $<0.001$. 
A
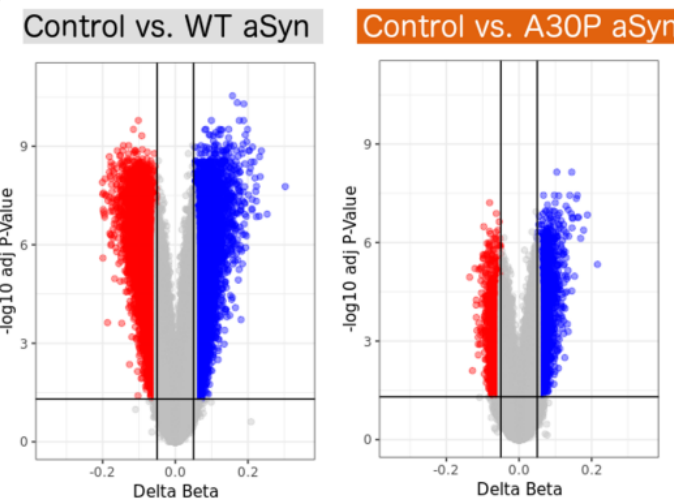

c

Enrichment/depletion of hits by genomic context

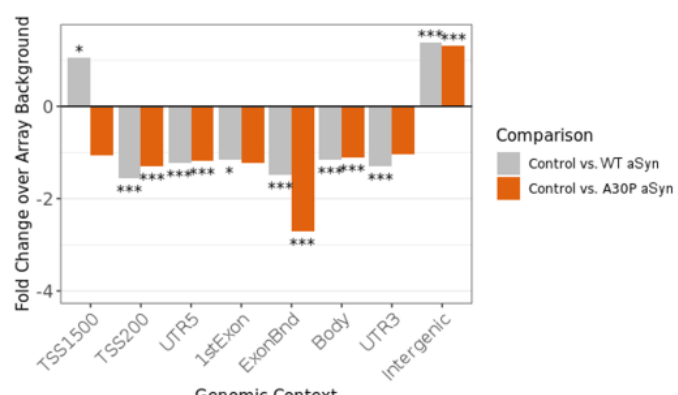

Genomic Context

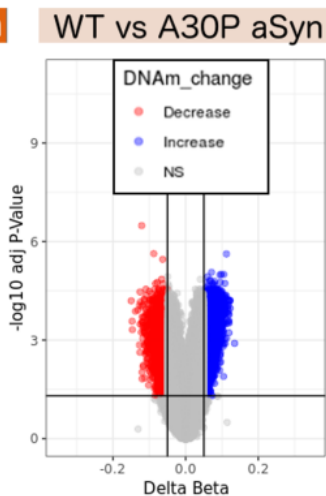

Enrichment/depletion of hits by relation to $\mathrm{CpG}$ island

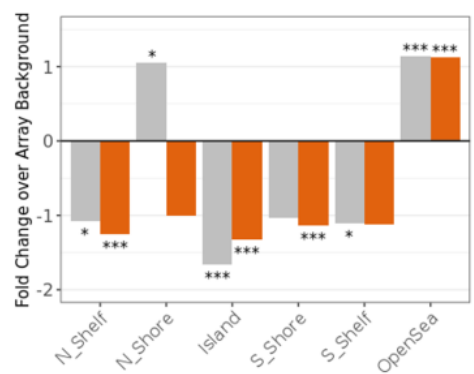

Genomic Context
B

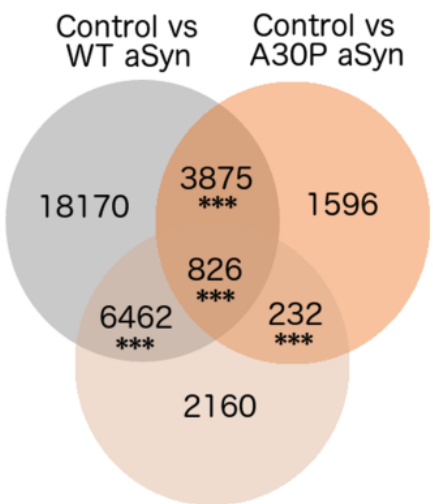

WT vs A30P aSyn

Figure 2. Overexpression of WT and A30P mutant aSyn altered the DNA methylome of dopaminergic neurons. (A) Volcano plots comparing DNAm patterns between control and WT aSyn LUHMES, control and A30P aSyn LUHMES, and WT aSyn and A30P aSyn LUHMES. Red: sites with decreased DNAm; blue: sites with increased DNAm (|delta beta| $>=0.05$ and padj $<=0.05$ ). (B) Number of differentially methylated probes unique to each comparison and probes shared between comparisons. ${ }^{*}$ padj $<0.05$, $* *$ padj $<0.005, * * *$ padj $<0.001$ (10,000 permutations). (C) Relative enrichment/depletion of differentially methylated sites across genomic contexts, permuted against array background. Grey: control vs. WT aSyn analysis; orange: control vs. A30P aSyn analysis. ${ }^{*}$ padj $<0.05, * *$ padj $<0.005, * * *$ by relation to CpG island, permuted against array background. Grey: control vs. WT aSyn analysis; orange: control vs. A30P aSyn analysis. 
A
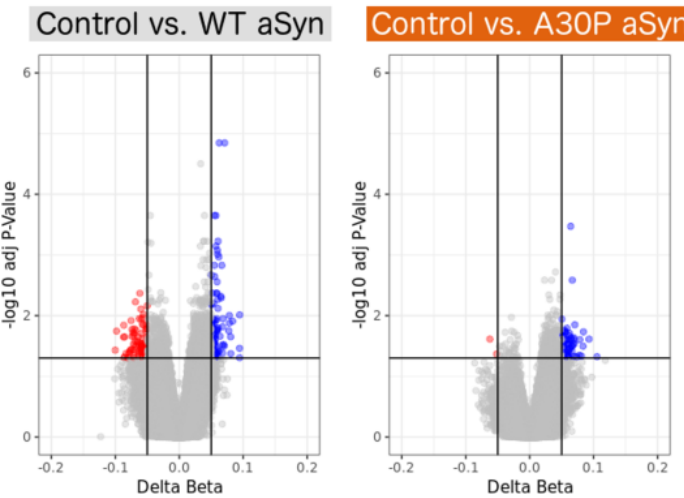

C

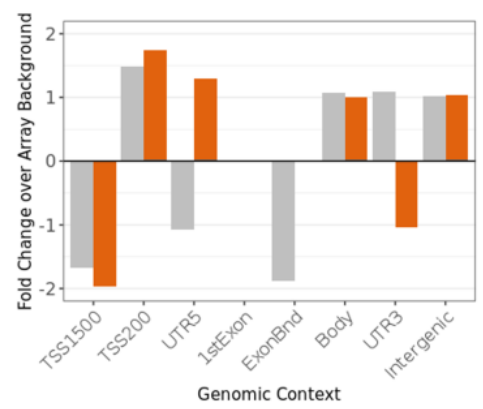

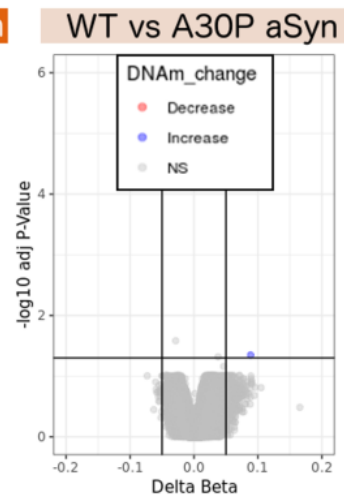

B

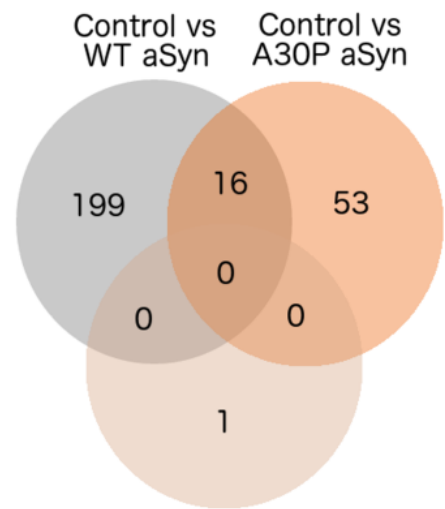

WT vs A30P aSyn

Figure 3. WT and A30P aSyn overexpression were correlated with increased DNA hydroxymethylation levels in dopaminergic neurons. (A) Volcano plots comparing DNAhm patterns between control and WT aSyn LUHMES, control and A30P aSyn LUHMES, and WT aSyn and A30P aSyn LUHMES. Red: sites with decreased DNAm; blue: sites with increased DNAm (|delta beta | >= 0.05 and padj <=0.05). (B) Number of differentially hydroxymethylated probes unique to each comparison and probes shared between comparisons. All padj > 0.05 (10,000 permutations). (C) Relative enrichment/depletion of differentially hydroxymethylated sites across genomic contexts, permuted against array background. Grey: control vs. WT aSyn analysis; orange: control vs. A30P aSyn analysis. (D) Relative enrichment/depletion of differentially hydroxymethylated sites by relation to $\mathrm{CpG}$ island, permuted against array background. Grey: control vs. WT aSyn analysis; orange: control vs. A30P aSyn analysis. * padj $<0.05(10,000$ permutations). 

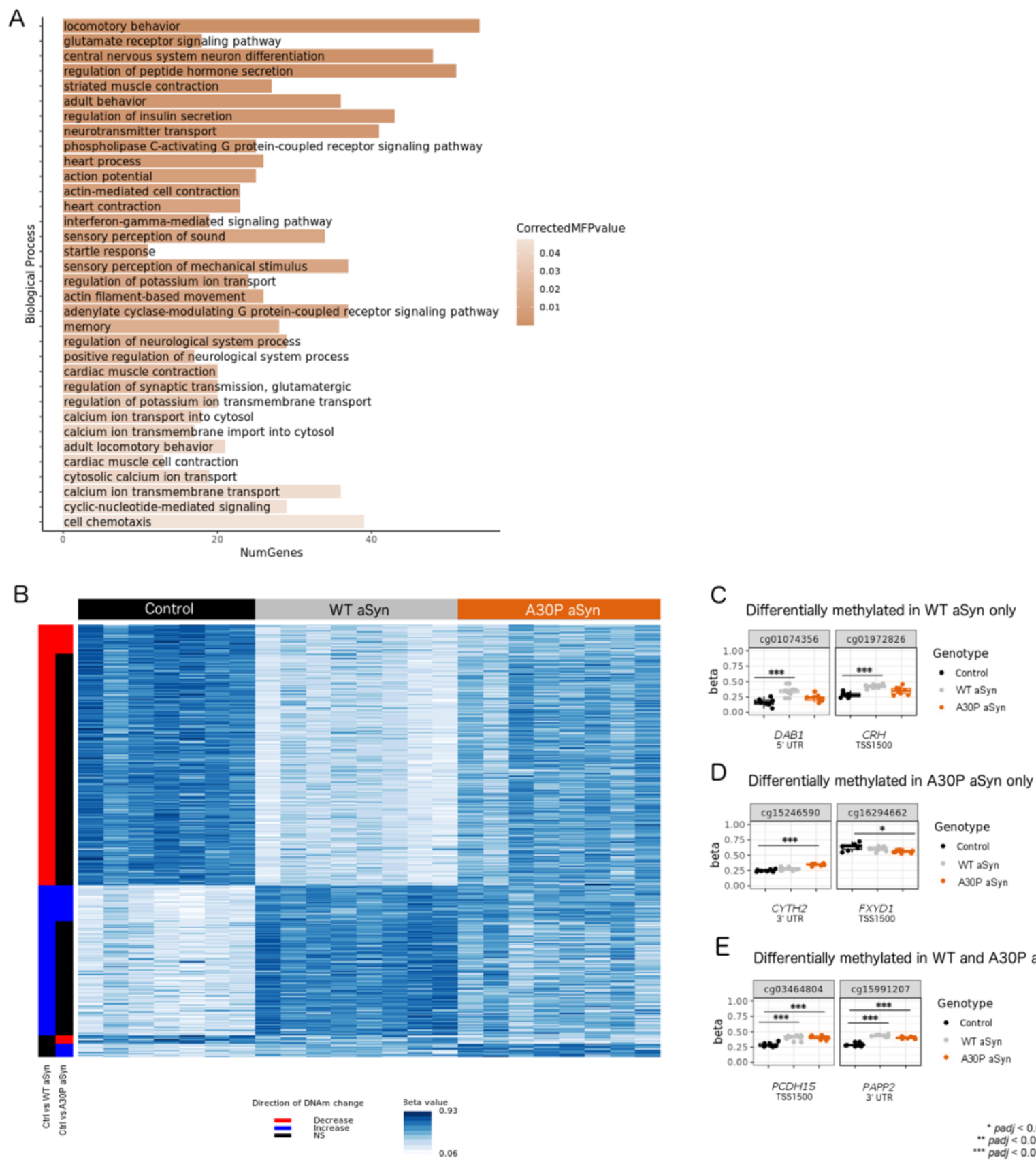

E Differentially methylated in WT and A30P aSyn

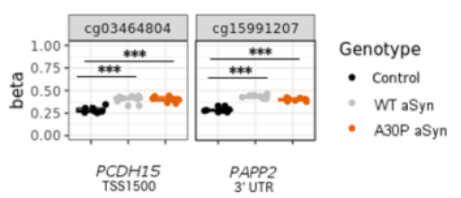

Figure 4. Differentially methylated genes in WT and A30P aSyn cells were enriched for locomotory behavior and glutamate receptor signaling functions. (A) GO biological process terms enriched at multifunctionality-corrected, multiple test-corrected $p$-value (CorrectedMFPvalue) $<0.05$ in genes shared between control vs. WT aSyn and control vs. A30P aSyn DNAm analyses. (B) Heat map showing beta values for all probes annotated to the "locomotory behavior" pathway and differentially methylated in at least one comparison. Row labels, left to right: Probes that passed significance thresholds ( $\mid$ delta beta| >= 0.05 and padj <= 0.05) in control vs WT aSyn comparison (decreased DNAm in WT aSyn: red; increased DNAm in WT aSyn: blue; non-significant: black). Probes that passed significance thresholds in control vs A30P aSyn comparison (decreased DNAm in A30P aSyn: red; 
bioRxiv preprint doi: https://doi org/10.1101/2021.06.12,448150; this version posted June 12, 2021. The copyright holder for this preprint (which was not certified by peer review) is the author/funder, who has granted bioRxiv a license to display the preprint in perpetuity. It is made available under aCC-BY-NC-ND 4.0 International license.

591 increased DNAm in A30P aSyn: blue; non-significant: black). (C) Representative examples of locomotory 592 behavior-related CpG sites differentially methylated only in WT aSyn cells. (D) Locomotory behavior-

593 related CpG sites differentially methylated only in A30P aSyn cells. (E) Examples of locomotory behavior594 related $\mathrm{CpG}$ sites differentially methylated in both genotypes. Black: control cells; grey: WT aSyn cells;

595 orange: A30P aSyn cells. ${ }^{*}$ padj $<0.05,{ }^{* *}$ padj $<0.005, * * *$ padj $<0.001$. 
bioRxiv preprint doi: https://doi.org/10.1101/2021.06.12.448150; this version posted June 12, 2021. The copyright holder for this preprint (which was not certified by peer review) is the author/funder, who has granted bioRxiv a license to display the preprint in perpetuity. It is made available under aCC-BY-NC-ND 4.0 International license.

A Control vs WT aSyn

Network built around GRIK2 Chi-square P-value $=1 \mathrm{e}-04$

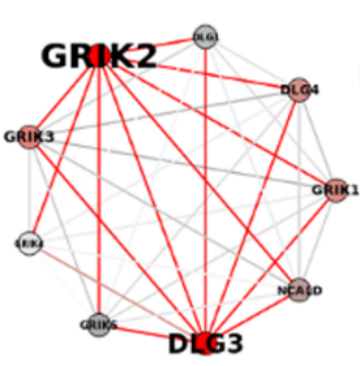

Chromosome 6
B Control vs A30P aSyn Network built around GRIK2:DLG3 Chi-square $P$-value $=2 \mathrm{e}-04$

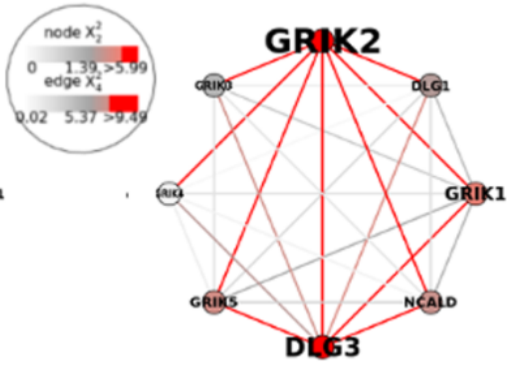

C GRIK2 mRNA expression

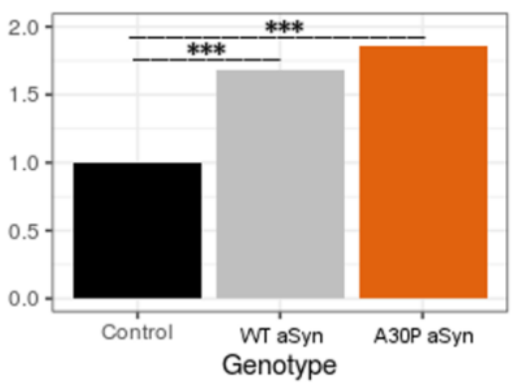

D
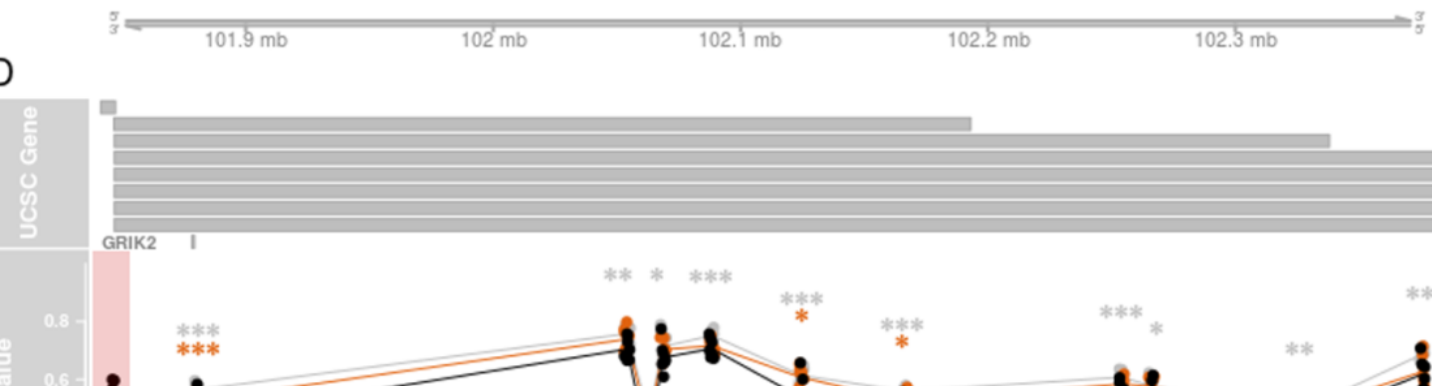

1

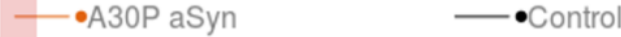

$\bullet$ WT aSyn

E

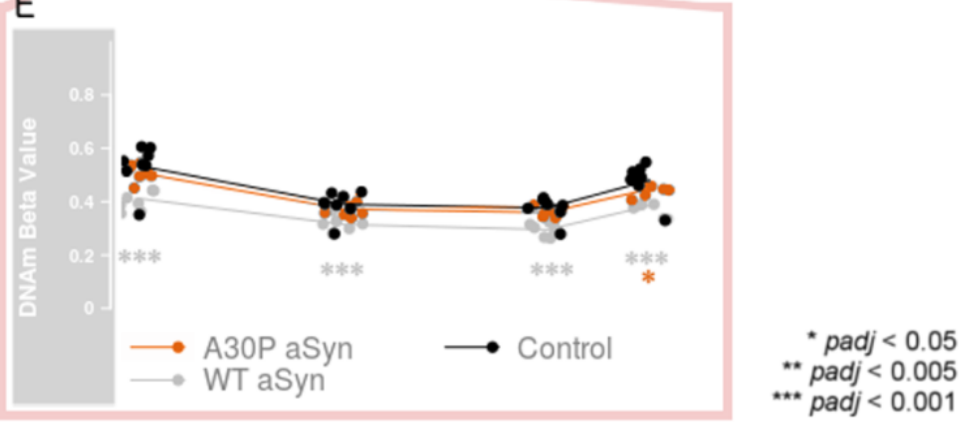

$\mathrm{F}$

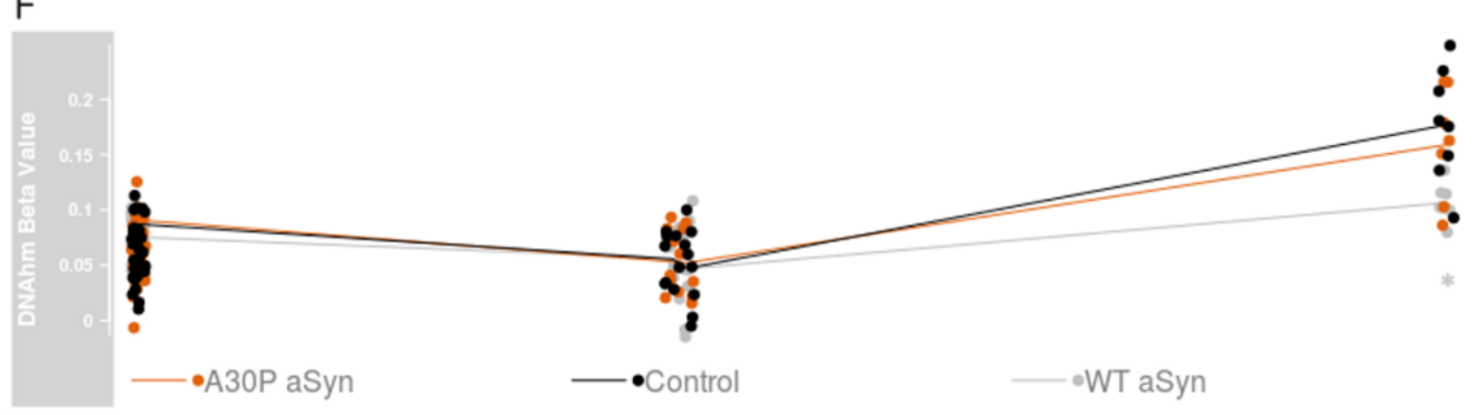


597 Figure 5. GRIK2 was differentially methylated, hydroxymethylated, and expressed in LUHMES

598 overexpressing either WT or A30P aSyn. (A) GRIK2 protein-protein interaction network which showed differential DNAm, DNAhm, and expression in control vs. WT aSyn cells for the underlying genes. (B)

600 GRIK2:DLG3 protein-protein interaction network which showed differential DNAm, DNAhm, and

601 expression in control vs. A30P cells for the underlying genes. (C) Relative expression of GRIK2 mRNA in

602 each genotype, normalized to control cells. (D) Top: UCSC hg19 coordinates are shown, with GRIK2

603 transcripts below. Bottom: DNAm beta values are shown for each sample, coloured by genotype. Black:

604 control; grey: WT aSyn; orange: A30P aSyn. (E) DNAm at the GRIK2 TSS200 region. (F) DNAhm levels for

605 EPIC array probes across the GRIK2 gene. * padj $<0.05, * *$ padj $<0.01, * * *$ padj $<0.005$.

606

607

608 